Research paper

\title{
Molecular and clinical studies in five index cases with novel mutations in the GLA gene
}

\author{
Carmela Zizzo $^{\mathrm{a}, *, 1}$, Ines Monte ${ }^{\mathrm{b}, 1}$, Antonio Pisani ${ }^{\mathrm{c}, 1}$, Pasquale Fatuzzo ${ }^{\mathrm{d}}$, Eleonora Riccio ${ }^{\mathrm{c}}$, \\ Margherita Stefania Rodolico e , Paolo Colomba ${ }^{\mathrm{a}}$, Maurizio Uva ${ }^{\mathrm{f}}$, Giuseppe Cammarata ${ }^{\mathrm{a}}$, Riccardo Alessandro ${ }^{\mathrm{a}, \mathrm{g}}$, \\ Francesco Iemolo ${ }^{\mathrm{a}}$, Giovanni Duro ${ }^{\mathrm{a}}$
}

a Institute of Biomedicine and Molecular Immunology (IBIM), National Research Council, Palermo, Italy

b Clinical Echocardiography, Research and Training European CVI Laboratory, Cardio-Thorax-Vascular Transplant Department, Policlinic “G. Rodolico" of University, Catania, Italy

${ }^{c}$ Department of Public Health, Section of Nephrology, Federico II University of Naples, Italy

d Internal Medicine, Nephrology and Dialysis Unit, Department of Clinical and Experimental Medicine, University of Catania, Italy

e Institute of Neurological Sciences (ISN), National Research Council, Catania, Italy

${ }^{\mathrm{f}}$ Operative Unit of Ophthalmology, University of Catania, Italy

${ }^{g}$ Department of Biopathology and Medical Biotechnology, University of Palermo, Italy

\section{A R T I C L E I N F O}

\section{Article history:}

Received 7 August 2015

Received in revised form 27 November 2015

Accepted 8 December 2015

Available online 12 December 2015

\section{Keywords:}

Fabry disease

Alpha-galactosidase A

c.846 847delTC

p.E341X

p.C382X

p.R227P

c. $639+5 G>T$

\begin{abstract}
A B S T R A C T
Fabry disease is a metabolic and lysosomal storage disorder caused by the functional defect of the $\alpha$-galactosidase A enzyme; this defect is due to mutations in the GLA gene, that is composed of seven exons and is located on the long arm of the X-chromosome (Xq21-22).

The enzymatic deficit is responsible for the accumulation of glycosphingolipids in lysosomes of different cellular types, mainly in those ones of vascular endothelium. It consequently causes a cellular and microvascular dysfunction.

In this paper, we described five novel mutations in the GLA gene, related to absent enzymatic activity and typical manifestations of Fabry disease. We identified three mutations (c.846_847delTC, p.E341X and p.C382X) that lead to the introduction of a stop codon in positions 297, 341 and 382. Moreover we found a missense mutation (p.R227P) in the exon 5 of the GLA gene and a single point mutation (c.639+5 G>T) occurring five base pairs beyond the end of the exon 4 . These mutations have never been found in our group of healthy control subjects $>2300$.
\end{abstract}

The studied patients presented some clinical manifestations, such as cornea verticillata, hypo-anhidrosis, left ventricular hypertrophy, cerebrovascular disorders and renal failure, that, considering the null enzymatic activity, suggest that the new mutations reported here are related to the classic form of Fabry disease.

The identification of novel mutations in patients with symptomatology referable to FD increases the molecular knowledge of the GLA gene and it gives clinicians an important support for the proper diagnosis of the disease.

(c) 2015 Elsevier B.V. All rights reserved.

\section{Introduction}

Fabry disease (FD) is a hereditary, X-linked, progressive and multisystemic lysosomal storage disease. It is a metabolic disorder caused by functional deficit of the $\alpha$-galactosidase A enzyme ( $\alpha$-GAL A) (Brady et al., 1967). This deficit is responsible for the alteration of the metabolism of some glycosphingolipids, mainly globotriaosylceramide (GB3) and globotriaosylsphingosine (Lyso-GB3), which consequently build up in

Abbreviations: FD, Fabry disease; $\alpha-G A L$ A, $\alpha$-galactosidase A; GB3, globotriaosylceramide; Lyso-GB3, globotriaosylsphingosine; GLA, GLA gene; ND, not determined; DGJ, 1-deoxygalactonojirimycin; ISE, intron splicing enhancer.

* Corresponding author at: Institute of Biomedicine and Molecular Immunology (IBIM), National Research Council,Via Ugo la Malfa 153, 90146 Palermo, Italy.

E-mail address: zizzo@ibim.cnr.it (C. Zizzo).

1 These authors contributed equally to the article. lysosomes of different cellular types, mostly in vascular endothelium cells (Desnick et al., 2001).

Clinical manifestations of the disease are slowly progressive, with a variable age of onset, severity, and course. Usually, it occurs during childhood or early adolescence manifesting angiokeratomas, lenticular and corneal opacity, microalbuminuria or proteinuria, and symptoms involving the peripheral nervous system. With the age, the disease leads to a gradually worsening of renal conditions and to cardiovascular involvement and cerebrovascular manifestations. These symptoms, or a combination of them, can cause the premature death of the patients during their fourth or fifth decade of life (Germain, 2010). Female patients, due to the random inactivation of the X-chromosome, usually present a milder and more variable symptomatology in comparison to male subjects (Dobrovolny et al., 2005). In addition to the classic form, atypical variants of Fabry disease have been described (Nakao et al., 
1995). These variants, that represent the $70 \%$ of Fabry patients, usually manifest a mild and late-onset phenotype and a non-specific symptomatology that make the diagnosis difficult. For this reason, the disease in these patients can be diagnosed in adulthood, when the irreversible impairment of internal organs already occurred (Lidove et al., 2012). Retrospective studies highlighted a delay in diagnosing FD in about $40 \%$ of male patients and $70 \%$ of female patients (Hoffmann and Mayatepek, 2009).

The disease is considered rare, with an incidence estimated about 1:40000 in general population. However, recent neonatal screening studies have shown a higher incidence: about 1 in 3100 among newborn males in Italy (Spada et al., 2006) and 1 in 1500 among newborn males in Taiwan (Hwu et al., 2009).

Considering its etiology, Fabry disease is a lysosomal enzymopathy caused by mutations in the GLA gene (GLA), encoding the $\alpha$-GAL A, located on the long arm of the X-chromosome (Xq21-22) (Germain, 2010). To date, more than 770 mutations in the coding GLA regions were described in Fabry patients (Human Gene Mutation Database, www.hgmd.org). However, there is not a clear genotype-phenotype correlation.

In this paper five mutations (c.846_847delTC, p.E341X, p.C382X, p.R227P, c.639 + 5G > T) found in the GLA gene of patients with clinical diagnosis of Fabry disease are described for the first time. The identification of novel mutations in patients with symptomatology referable to FD increases the molecular knowledge of the GLA gene and it gives clinicians an important support for the proper diagnosis of the disease. Moreover, the genetic investigation on the relatives of patients suffering from FD allows us to detect oligo- or pre-symptomatic subjects who can start as early as possible the specific therapy, before the organ involvement becomes irreversible.

\section{Material and methods}

\subsection{Patient specimens}

Peripheral blood was collected, using EDTA as an anticoagulant, from patients with clinical manifestations related to FD symptomatology. The study was approved by the Hospital Ethics Committee of University of Palermo, and signed informed consent was obtained from all participants.

\subsection{DNA isolation}

DNA samples were isolated from whole blood by column extraction (GenElute Blood Genomic DNA Kit, Miniprep, Sigma-Aldrich, USA), and their concentrations were determined using a spectrophotometer.

\subsection{High resolution melting analysis and DNA sequencing}

High resolution melting analysis was performed on DNA samples using the Light Cycler 480 system (Roche Applied Science, Germany). Seven pairs of primers were designed for the analysis of seven target regions containing the seven exons of the GLA gene and the regulatory sequences flanking them. In order to ensure the detection of hemizygotes, male subjects were analyzed by adding a wild type DNA counterpart, in a 1:1 quantity ratio, to their DNA sample to produce artificial heterozygotes and induce heteroduplex formation.

PCR products presenting melting curves different in position or shape from those of the wild type control were purified and sequenced to identify suspected mutations, using automated DNA sequencer at BMR Genomics.

\subsection{Alpha-galactosidase activity assay}

Alpha-galactosidase A activity was determined by the Dried Blood Filter Paper test described by Chamoles et al. (2001), with the following modifications: spot of $5.5 \mu \mathrm{l}$ of blood in $6-\mathrm{mm}$ diameter circle paper was placed into a 96-well plate suitable for fluorimetric assays and incubated for $16 \mathrm{~h}$ at $37^{\circ} \mathrm{C}$ in a thermomixer; the reaction was terminated by the addition of $250 \mu \mathrm{l}$ of $0.1 \mathrm{~mol} / \mathrm{l}$ ethylenediamine ( $\mathrm{pH} 11.4$ ). The fluorescence of background which is not due to the specific enzymatic activity, was determined for each sample, doing another reaction in the presence of $0.15 \mathrm{nmol} / 1$ 1-deoxygalactonojirimycin (DGJ, the inhibitor of alpha galactosidase A) in $0.15 \mathrm{~mol} / \mathrm{l}$ citrate phosphate buffer ( $\mathrm{pH}$ 4.4). This background was subtracted from the fluorescence of the sample.

\section{Results}

In this paper, we describe five novel mutations that we found in patients with clinical diagnosis of Fabry disease, in whom we performed molecular analyses (Table 1). These alterations are not present in the Human Gene Mutation Database (http://www.hgmd.org) and in Fabry disease databases. We named the five mutations according to the guidelines for mutation nomenclature recommended by the Human Genome Variation Society (www.hgvs.org/mutnomen).

Where it was possible, we also extended the genetic and enzymatic analyses of alpha galactosidase A to the relatives of probands (Table 2).

\subsection{Index case 1}

Patient 1 is a 24-year-old girl that manifested angiokeratomas and burning pain in hands and feet since childhood. Fabry disease was suspected after that the clinician found a left ventricular wall thickening typical of Fabry disease.

\subsubsection{Molecular diagnosis (index case 1)}

The genetic analysis of the GLA gene in this patient allowed us to identify a new two base pair deletion (c.846_847delTC) in the exon 6 of the gene. This small deletion causes a frameshift causing a stop codon at position 297 resulting in a protein of 296 amino acids rather than of 429; the DBFP test showed no enzymatic activity $(0.3 \mathrm{nmol} / \mathrm{h} / \mathrm{ml})$.

Table 1

Clinical and molecular data of the five index cases. In bold, values below the normal range. Normal values of $\alpha$-galactosidase A activity assayed in whole blood are $>3.0 \mathrm{nmol} / \mathrm{h} / \mathrm{ml}$.

\begin{tabular}{|c|c|c|c|c|c|c|c|c|c|}
\hline $\begin{array}{l}\text { Index } \\
\text { case }\end{array}$ & Gender/age & $\begin{array}{l}\text { GLA } \\
\text { mutation }\end{array}$ & $\begin{array}{l}\text { Enzymatic } \\
\text { activity }\end{array}$ & Acroparesthesias & Angiokeratomas & $\begin{array}{l}\text { Cardiac } \\
\text { manifestations }\end{array}$ & $\begin{array}{l}\text { Renal } \\
\text { manifestations }\end{array}$ & $\begin{array}{l}\text { Neurological } \\
\text { manifestations }\end{array}$ & Other signs \\
\hline 1 & $\mathrm{~F} / 24$ & c.846_847del AG & 0.3 & + & + & + & - & - & - \\
\hline 2 & $\mathrm{~F} / 62$ & p.E341X & 1.6 & - & + & + & + & + & Cornea verticillata \\
\hline 3 & $\mathrm{~F} / 44$ & p.C382X & 3.6 & + & - & + & + & + & $\begin{array}{l}\text { Cornea verticillata, fatigue, } \\
\text { gastrointestinal manifestations, } \\
\text { recurrent fevers }\end{array}$ \\
\hline 4 & $\mathrm{~F} / 59$ & p.R227P & 2.3 & - & - & + & + & - & Anhidrosis \\
\hline 5 & $\mathrm{M} / 43$ & c. $639+5 G>T$ & 0.2 & + & + & + & + & - & $\begin{array}{l}\text { Recurrent fever, heat and cold } \\
\text { intolerance, hypohidrosis, fatigue }\end{array}$ \\
\hline
\end{tabular}




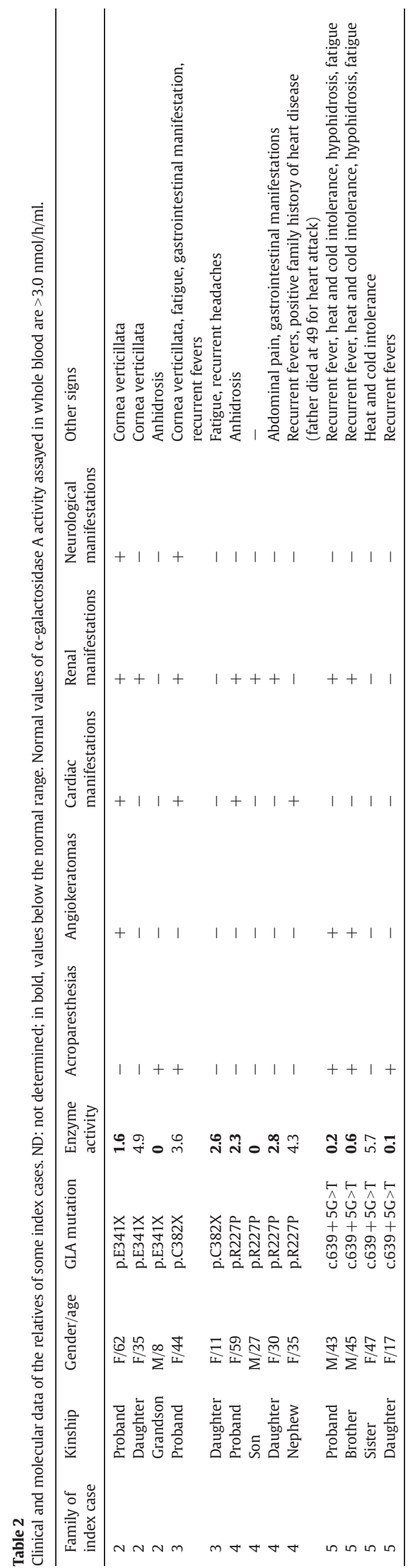

\subsection{Index case 2}

Patient 2 is a 62-year-old woman, affected by hypertension, diabetes and chronic renal failure. She experienced two strokes in three years and she underwent several cardiovascular controls for atrial fibrillation and hypertensive heart disease: these highlighted a hypertrophic cardiomyopathy. She also manifested cornea verticillata in the right eye and cataract in the left one and she showed angiokeratomas of the vulvar region. All these clinical data led clinicians to hypothesize the diagnosis of Fabry disease (Table 2).

In addition, her 35-year-old daughter showed early signs of cornea verticillata in both eyes and microalbuminuria; cardiovascular and dermatologic examinations resulted negative. The proband's grandson is a child aged 8 who was apparently asymptomatic, though he showed anhidrosis and acroparesthesias, peculiar manifestations of Fabry disease in pediatric age (Table 2).

\subsubsection{Molecular diagnosis (index case 2)}

The clinical suspect of Fabry disease encouraged us to perform the molecular analyses in this patient and her relatives (Table 2). The genetic analysis of GLA revealed a novel non-sense mutation (c.1021G $>$ T). It is a $\mathrm{G}>\mathrm{T}$ transversion at position 1021 of the GLA cDNA that introduces a stop codon at position 341 resulting in a truncated protein (p.E341X). Enzymatic activity resulted $1.6 \mathrm{nmol} / \mathrm{h} / \mathrm{ml}$ in the proband, below normal values of $\alpha$-galactosidase A activity. We extended the molecular study to other relatives and we found the same mutation in proband's daughter (her enzymatic activity was in the normal range) and in one of the two grandchildren, who showed 0 activity (Table 2).

\subsection{Index case 3}

Patient 3 is a 44-year-old woman, who came to our examination after the casual finding of proteinuria and albuminuria. The woman showed a clinical history of acroparesthesias, fatigue, recurrent fevers and gastrointestinal manifestations in childhood, and she had manifested for some years cardiac arrhythmia, hearing loss, tinnitus and recurrent headaches. Moreover, she had received an initial diagnosis of rheumatoid arthritis. After the clinical suspect of Fabry disease, clinicians performed ocular examination that revealed cornea verticillata, typical of Fabry disease (Table 2).

\subsubsection{Molecular diagnosis (index case 3 )}

The genetic analysis of GLA revealed a novel non-sense mutation in the exon 7 of the gene (c.1146C $>A$ ). It is a $C>A$ transversion at position 1146 of the GLA cDNA that introduces a stop codon at position 382 resulting in a truncated protein (p.C382X). The analysis of enzymatic activity was $3.6 \mathrm{nmol} / \mathrm{h} / \mathrm{ml}$, just above the normal range (normal values of alpha galactosidase A activity assayed in whole blood are $>3.0 \mathrm{nmol} / \mathrm{h} /$ $\mathrm{ml}$ ). This is plausible considering that this patient is a female. We also performed the genetic and enzymatic study in the 11-year-old proband's daughter, who manifested fatigue and recurrent headaches (Table 2). We found the same mutation of her mother and the enzymatic activity resulted $2.6 \mathrm{nmol} / \mathrm{h} / \mathrm{ml}$, below the normal range.

\subsection{Index case 4}

Patient 4 is a 59-year-old woman with modest proteinuria and left ventricular hypertrophy. Moreover, she reported anhidrosis, which appeared in childhood. The patient has a son (27 years old) and a daughter ( 30 years old): the son underwent dialysis after the incidental finding of impaired renal function; the daughter suffered from abdominal pain and gastrointestinal manifestations, and she also reported mild proteinuria. Moreover, the patient's brother died at the age of 49 for acute myocardial infarction (Table 2). 


\subsubsection{Molecular diagnosis (index case 4)}

After the clinical suspect of Fabry disease, we performed the analysis of seven exons of the GLA gene and the intronic regions flanking them. We found the novel mutation c.680G $>$ C that causes the arginine to proline substitution at amino acid 227 of the protein (p.R227P). Enzymatic activity resulted $2.3 \mathrm{nmol} / \mathrm{h} / \mathrm{ml}$ (normal values of $\alpha$-galactosidase A activity in whole blood are $>3.0 \mathrm{nmol} / \mathrm{h} / \mathrm{ml})$.

We also extended the molecular study to other members of family (Table 2). Three other family members showed the same mutation: the patient's son (enzymatic activity $0 \mathrm{nmol} / \mathrm{h} / \mathrm{ml}$ ), the patient's daughter (enzymatic activity $2.8 \mathrm{nmol} / \mathrm{h} / \mathrm{ml}$ ) and also the patient's niece, daughter of the patient's brother who died at the age of 49 for acute myocardial infarction. In this last young woman, the enzymatic activity resulted in the normal range $(4.3 \mathrm{nmol} / \mathrm{h} / \mathrm{ml})$, although she already manifested slight left ventricular hypertrophy and recurrent fevers arisen in early age.

\subsection{Index case 5}

Patient 5 is a 43-year-old man. After the incidental finding of severe renal failure, he underwent peritoneal dialysis for 4 years; then, he underwent kidney transplantation. The 45-year-old brother of this patient had a similar clinical history: renal failure of unknown cause and subsequent kidney transplantation. Both brothers showed a clinical history of recurrent fevers in childhood, acroparesthesias, intolerance to heat and cold, hypohidrosis and fatigue.

They also had "angiocheratoma diffusum" since youth. Considering the typical clinical history and medical records, Fabry disease was investigated (Table 2). The evaluation of the disease was extended to their 47-year-old sister, who manifested only intolerance to heat and cold, and the 17-year-old proband's daughter, who had recurrent fever and acroparesthesias.

\subsubsection{Molecular diagnosis (index case 5)}

Molecular studies showed a novel point mutation (c.639+5G>T) in the intron-exon splice junction, 5 base pairs downstream from the end of exon 4 of the GLA gene; enzymatic activity was absent $(0.2 \mathrm{nmol} / \mathrm{h} / \mathrm{ml})$ in the proband.

The genetic and enzymatic analyses were extended to other family members and, among these, three patients with the same mutation were identified (Table 2). The three affected subjects were: the proband's brother, who had $0.6 \mathrm{nmol} / \mathrm{h} / \mathrm{ml}$ enzymatic activity, the proband's daughter, who had $0.1 \mathrm{nmol} / \mathrm{h} / \mathrm{ml}$ enzymatic activity; the proband's sister, who showed normal values of $\alpha$-galactosidase A activity.

\section{Discussion}

In this paper, we described five novel mutations (c.846_847delTC, p.E341X, p.C382X, p.R227P, c.639+5G > T) that we found in unrelated patients, confirming the genetic heterogeneity observed in subjects affected by Fabry disease. These mutations are not present in the Human Gene Mutation Database (http://www.hgmd.org) and in Fabry disease databases. Where it was possible, we also extended the genetic and enzymatic analyses of alpha galactosidase A to the relatives of probands.

The mutations responsible for the introduction of a premature stop codon (c.846_847delTC, p.E341X, p.C382X) were found in patients affected by the classical phenotype of Fabry disease. This is plausible because these mutations result in truncated and non-functional proteins. In fact, the resulting proteins lack almost one third, one fifth and one eighth of the wild-type protein of 429 amino acids, respectively.

The loss of function in these proteins was also proved by the absent enzymatic activity, observed in the only male with a mutation causing a stop codon. He is an 8-year-old child, grandson of the index case 2 , who was affected by p.E341X mutation and manifested anhidrosis and acroparesthesias. The other subjects with mutations resulting in a stop codon, who were all females, had values of enzymatic activity below the normal range (three of them) or normal (two of them). This variability is due to the chromosome $\mathrm{X}$ inactivation. Thus, on the basis of which chromosome is silenced, (mutated one or wt one) the resulting protein will be a functional or an inactive enzyme. Moreover, until a few years ago the inactivation of the $\mathrm{X}$ chromosome was thought to be random, but today this concept has been revised. It was observed that in some females, the $\mathrm{X}$ chromosome inactivation is not random in all tissues but some of them show a skewed inactivation to the wild type $\mathrm{X}$ chromosome or the mutated one - phenomenon that, still today, is without an explanation (Echevarria et al., 2015). This results in a great clinical heterogeneity ranging from the absence of the manifestations to a severity comparable to the one of the males affected by Fabry disease (Whybra et al., 2001; MacDermot et al., 2001; Wilcox et al., 2008).

The missense mutation described in this article is also responsible of Fabry phenotype. Patient 4 with p.R227P mutation was a 59-year-old woman. Although the advanced age, she presented only a mild proteinuria and a marked left ventricular hypertrophy, but she had a family history strongly suggestive of Fabry disease: her brother died at the age of 49 for acute myocardial infarction.

Moreover, according to Polyphen 2 (http://genetics.bwh.harvard. edu/pph2), a tool that predicts possible impact of an amino acid substitution on the structure and function of a human protein, the mutation p.R227P is probably damaging. It also predicts that R227 is highly conserved throughout the evolution (Fig. 1). The amino acid is not localized in the binding site or in the catalytic domain, however it is closely near the latter. In Fabry database (http://fabry-database.org/), a different mutation affecting this amino acid (p.R227Q) is predicted to cause the classical form of Fabry disease, probably because of a structural change on active site residues. Moreover mutations in other amino acids flanking R227 (p.W226R, p.N224S and p.N224D) are responsible for classic phenotype of Fabry disease (Ashton-Prolla et al., 2000; Guffon et al., 1998). These data, in addition to the null activity that we found in a male with p.R227P, confirm the pathogenic role of this mutation.

Moreover, in this paper, we describe a family, the proband and his three relatives with the c. $639+5 \mathrm{G}>\mathrm{T}$ mutation. It is a $\mathrm{G}>\mathrm{T}$ transition occurring 5 base pairs downstream from the end of exon 4 of the GLA gene, in a regulation splicing site. The subjects, in whom this mutation was found, manifested typical signs and symptoms of Fabry disease, such as acroparesthesias, heat and cold intolerance, recurrent fever, angiokeratomas and renal failure. Moreover, the enzyme activity resulted absent or strongly reduced in three out of four subjects, instead in a female with the same mutation, because of X-chromosome inactivation, the enzyme activity was normal. This mutation is not in the coding region of the GLA gene but it is the only mutation that we found in and no enzyme activity. It was never found in more than 2300 healthy control subjects.



Fig. 1. Alignment of wild-type human $\alpha$-Gal A amino acids with other organisms sequences. The rectangle indicates the amino acid at position 227 that we found mutated in the index case. 
Using RegRNA 2.0 (http://regrna2.mbc.nctu.edu.tw/), a computational platform that is able to find hypothetical regulatory sites in a DNA sequence, we found that the mutation occurs in a putative site of splicing regulation. The guanine in position c. $639+5$ of the GLA gene is included in a sequence of 5 bases that is predicted to be a putative intron splicing enhancer (ISE sequence) by RegRNA program. The G>T substitution in this position would cause the loss of function of ISE, because of the modification of this sequence. Mutations in these sites could play a role in the synthesis of GLA mRNA and in the (qualitative and quantitative) production of $\alpha$-galactosidase $A$. It is well known in literature that the alternative splicing products of mRNAs may be located in different cell types. It was estimated that the aberrant splicing of mRNAs causes the $15 \%$ of human genetic diseases (Tazi et al., 2009).

All the patients studied in this paper, showed symptoms referable to the classical form of Fabry disease with first manifestations, such as acroparesthesias, fatigability, gastrointestinal manifestations and hypo-anhidrosis in childhood. However, all the index cases were diagnosed in adulthood when one or more appreciable clinical manifestations such as ictus, renal failure and left ventricular hypertrophy led the clinicians to study Fabry disease.

The main reasons of the missed diagnoses of Fabry disease are due to the phenotypic variability - both between related patients and between unrelated ones. Secondly, several clinical features of Fabry disease are usually present in the general population, such as neuropathic and abdominal pain, headache, tinnitus, hearing loss, gastrointestinal manifestations, renal and cardiovascular diseases (Ries and Gal, 2006).

\section{Conclusion}

The genotype-phenotype correlation is still unclear in Fabry disease. In fact, some mutations can cause different phenotypes both between related subjects and between unrelated patients, suggesting the existence of genetic and/or environmental factors that could affect the severity of the disease. This correlation is also complicated by several factors such as the high number of private mutations and the phenotypic heterogeneity associated to the same mutation. Moreover, patients with Fabry disease can develop symptoms related to the disease that are observed with high prevalence in the general population (Ries and Gal, 2006; Schäfer et al., 2005).

Considering the clinical point of view, Fabry disease should be considered in patients with atypical clinical course, uncertain diagnosis, or clinical picture with systemic involvement that is not clear, in particular when a clinical family history is positive. This clinical approach would help to limit the misdiagnosis between Fabry disease and other systemic disorders that are more common. Moreover, it could contribute to reduce the time elapsing between the onset of the first symptoms and the proper diagnosis of Fabry disease, avoiding useless treatments for the patients and starting the therapy for the disease that to date is available. The data in literature show the importance of specific therapeutic intervention made as early as possible, before the organ involvement becomes irreversible (Pisani et al., 2014, 2012, 2015, 2005; Spinelli et al., 2004).

\section{Acknowledgments}

The financial support for the conduct of the research and preparation of the article was provided by the National Research Council (CNR) (ME.P01.014.008).

\section{References}

Ashton-Prolla, P., Tong, B., Shabbeer, J., Astrin, K.H., Eng, C.M., Desnick, R.J., 2000 Fabry disease: twenty-two novel mutations in the alpha-galactosidase A gene and genotype/phenotype correlations in severely and mildly affected hemizygotes and heterozygotes. J. Investig. Med. 48 (4), 227-235.

Brady, R.O., Gal, A.E., Bradley, R.M., Martensson, E., Warshaw, A.L., Laster, L., 1967. Enzymatic defect in Fabry's disease. Ceramidetrihexosidase deficiency. N. Engl. J. Med. 276 (21), 1163-1167.

Chamoles, N.A., Blanco, M., Gaggioli, D., 2001. Fabry disease: enzymatic diagnosis in dried blood spots on filter paper. Clin. Chim. Acta 308 (1-2), 195-196.

Desnick, R.J., Ioannou, Y.A., Eng, C.M., 2001. Alpha-galactosidase A deficiency: Fabry disease. In: Scriver, C.R., Beaudet, A.L., Sly, W.S., Valle, D. (Eds.), The Metabolic and Molecular Basis of Inherited Disease, eighth ed. McGraw-Hill, New York, pp. 3733-3774.

Dobrovolny, R., Dvorakova, L., Ledvinova, J., Magage, S., Bultas, J., Lubanda, J.C., Elleder, M., Karetova, D., Pavlikova, M., Hrebicek, M., 2005. Relationship between X-inactivation and clinical involvement in Fabry heterozygotes. Eleven novel mutations in the alphagalactosidase A gene in the Czech and Slovak population. J. Mol. Med. 83 (8), 647-654

Echevarria, L., Benistan, K., Toussaint, A., Dubourg, O., Hagege, A.A., Eladari, D., Jabbour, F. Beldjord, C., De Mazancourt, P., Germain, D.P., 2015. X-chromosome inactivation in female patients with Fabry disease. Clin Genet http://dx.doi.org/10.1111/cge.12613 (Epub ahead of print).

Germain, D.P., 2010. Fabry disease. Orphanet J. Rare Dis. 5, 30.

Guffon, N., Froissart, R., Chevalier-Porst, F., Maire, I., 1998. Mutation analysis in 11 French patients with Fabry disease. Hum Mutat (Suppl. 1), S288-S290.

Hoffmann, B., Mayatepek, E., 2009. Fabry disease - often seen, rarely diagnosed. Dtsch. Arztebl. Int. 106 (26), 440-447.

Hwu, W.L., Chien, Y.H., Lee, N.C., Chiang, S.C., Dobrovolny, R., Huang, A.C., Yeh, H.Y., Chao, M.C., Lin, S.J., Kitagawa, T., Desnick, R.J., Hsu, L.W., 2009. Newborn screening for Fabry disease in Taiwan reveals a high incidence of the later-onset GLA mutation c. $936+919 G>A$ (IVS4 + 919G $>$ A). Hum. Mutat. 30 (10), 1397-1405.

Lidove, O., Kaminsky, P., Hachulla, E., Leguy-Seguin, V., Lavigne, C., Marie, I., Maillot, F. Serratrice, C., Masseau, A., Chérin, P., Cabane, J., Noel, E., FIMeD investigators, 2012. Fabry disease 'The New Great Imposter': results of the French Observatoire in Internal Medicine Departments (FIMeD). Clin. Genet. 81 (6), 571-577.

MacDermot, K.D., Holmes, A., Miners, A.H., 2001. Anderson-Fabry disease: clinical manifestations and impact of disease in a cohort of 60 obligate carrier females. J. Med. Genet. 38 (11), 769-775.

Nakao, S., Takenaka, T., Maeda, M., Kodama, C., Tanaka, A., Tahara, M., Yoshida, A. Kuriyama, M., Hayashibe, H., Sakuraba, H., Tanaka, H., 1995. An atypical variant of Fabry's disease in men with left ventricular hypertrophy. N. Engl. J. Med. 333 (5), 288-293.

Pisani, A., Spinelli, L., Sabbatini, M., Andreucci, M.V., Procaccini, D., Abbaterusso, C., Pasquali, S., Savoldi, S., Comotti, C., Cianciaruso, B., 2005. Enzyme replacement therapy in Fabry disease patients undergoing dialysis: effects on quality of life and organ involvement. Am. J. Kidney Dis. 46 (1), 120-127.

Pisani, A., Visciano, B., Roux, G.D., Sabbatini, M., Porto, C., Parenti, G., Imbriaco, M., 2012. Enzyme replacement therapy in patients with Fabry disease: state of the art and review of the literature. Mol. Genet. Metab. 107 (3), 267-275.

Pisani, A., Visciano, B., Imbriaco, M., Di Nuzzi, A., Mancini, A., Marchetiello, C., Riccio, E., 2014. The kidney in Fabry's disease. Clin. Genet. 86 (4), 301-309.

Pisani, A., Sabbatini, M., Duro, G., Colomba, P., Riccio, E., 2015. Antiproteinuric effect of add-on paricalcitol in Fabry disease patients: a prospective observational study. Nephrol. Dial. Transplant. 30 (4), 661-666.

Ries, M., Gal, A., 2006. Genotype-phenotype correlation in Fabry disease. In: Mehta, A. Beck, M., Sunder-Plassmann, G. (Eds.), Fabry Disease: Perspectives From 5 Years of FOS. Oxford PharmaGenesis, Oxford (Chapter 34).

Schäfer, E., Baron, K., Widmer, U., Deegan, P., Neumann, H.P., Sunder-Plassmann, G., Johansson, J.O., Whybra, C., Ries, M., Pastores, G.M., Mehta, A., Beck, M., Gal, A., 2005. Thirty-four novel mutations of the GLA gene in 121 patients with Fabry disease. Hum. Mutat. 25 (4), 412

Spada, M., Pagliardini, S., Yasuda, M., Tukel, T., Thiagarajan, G., Sakuraba, H., Ponzone, A. Desnick, R.J., 2006. High incidence of later-onset Fabry disease revealed by newborn screening. Am. J. Hum. Genet. 79 (1), 31-40.

Spinelli, L., Pisani, A., Sabbatini, M., Petretta, M., Andreucci, M.V., Procaccini, D., Lo Surdo N., Federico, S., Cianciaruso, B., 2004. Enzyme replacement therapy with agalsidase beta improves cardiac involvement in Fabry's disease. Clin Genet 66 (2), 158-165.

Tazi, J., Bakkour, N., Stamm, S., 2009. Alternative splicing and disease. Biochim. Biophys. Acta 1792 (1), 14-26.

Whybra, C., Kampmann, C., Willers, I., Davies, J., Winchester, B., Kriegsmann, J., Brühl, K., Gal, A., Bunge, S., Beck, M., 2001. Anderson-Fabry disease: clinical manifestations of disease in female heterozygotes. J. Inherit. Metab. Dis. 24 (7), 715-724.

Wilcox, W.R., Oliveira, J.P., Hopkin, R.J., Ortiz, A., Banikazemi, M., Feldt-Rasmussen, U., Sims, K., Waldek, S., Pastores, G.M., Lee, P., Eng, C.M., Marodi, L., Stanford, K.E., Breunig, F., Wanner, C., Warnock, D.G., Lemay, R.M., Germain, D.P., 2008. Fabry registry. Females with Fabry disease frequently have major organ involvement: lessons from the Fabry registry. Mol. Genet. Metab. 93 (2), 112-128. 